\title{
Investigation of interaction
}

between cellulase and residual lignin fractions in acid-pretreated bamboo residues and their effect on enzymatic digestibility

\author{
Wenqian Lin \\ Nanjing Forestry University \\ Jinlai Yang \\ China National Bamboo Research Center \\ Yayue Zheng \\ Nanjing Forestry University \\ Caoxing Huang ( $\square$ hcx@njfu.edu.cn ) \\ Nanjing Forestry University \\ Qiang Yong \\ Nanjing Forestry University
}

\section{Research}

Keywords: dilute acid pretreatment, enzymatic digestibility, lignin, SPR technique, interaction mechanism

Posted Date: April 1st, 2021

DOI: https://doi.org/10.21203/rs.3.rs-371092/v1

License: (c) (i) This work is licensed under a Creative Commons Attribution 4.0 International License.

Read Full License 


\section{Investigation of interaction between cellulase and residual lignin fractions in acid-pretreated bamboo residues and their effect on enzymatic digestibility}

Wenqian Lin ${ }^{\mathrm{a}, 1}$, Jinlai Yang ${ }^{\mathrm{b}, 1}$, Yayue Zheng ${ }^{\mathrm{a}}$, Caoxing Huang a,", Qiang Yong a

Affiliation:

${ }^{a}$ Jiangsu Co-Innovation Center of Efficient Processing and Utilization of Forest Resources, College of Chemical Engineering, Nanjing Forestry University, Nanjing 210037, China

${ }^{\mathrm{b}}$ China National Bamboo Research Center and Key Laboratory of High Efficient Processing of Bamboo of Zhejiang Province, Hangzhou 310012, Zhejiang, China.

* Corresponding author:

Tel: +86 25 85427587; E-mail address: hcx@njfu.edu.cn

1: Wenqian Lin and Jinlai Yang contributed equally to this work, regarding as the first author 


\section{ABSTRACT}

Background: During dilute acid pretreatment, pseudo lignin and lignin form droplets which deposit on the surface of lignocellulose, and further inhibit its enzymatic hydrolysis. However, how this lignin interacts with cellulase enzymes and then affects enzymatic hydrolysis is still unknown. In this work, different fractions of surface lignin (SL) obtained from dilute acid pretreated bamboo residues (DAP-BR) were extracted by various organic reagents and the residual lignin in extracted DAP-BR was obtained by milled wood lignin (MWL) method. All the obtained lignin fractions from DAP-BR were used to investigate the interaction mechanism between lignin and cellulase using surface plasmon resonance (SPR) technology in order to understand how they affect enzymatic hydrolysis

Results: Results showed that removing surface lignin significantly decrease the enzymatic hydrolysis of DAP-BR from $36.5 \%$ to $18.6 \%$. The addition of MWL samples to Avicel decreased enzymatic hydrolysis of Avicel, while different SL samples showed a slight increase to its enzymatic digestibility. Due to the higher molecular weight and hydrophobicity of MWL samples versus the SL samples, stronger affinity for MWL (KD $=6.8-24.7 \mathrm{nM})$ was found versus that of SL $(\mathrm{KD}=39.4-52.6 \mathrm{nM})$ by SPR analysis. The affinity constant of all tested lignin had good correlations $\left(\mathrm{R}^{2}>0.6\right)$ with their effects on enzymatic digestibility of extracted DAP-BR and Avicel.

Conclusions: This work reveals that the surface lignin on DAP-BR is necessary towards 
maintaining enzyme digestibility levels, and its removal has a negative impact on the substrate's digestibility.

Keywords: dilute acid pretreatment; enzymatic digestibility; lignin; SPR technique; interaction mechanism

\section{Background}

Growing population and related energy demand for societies across the world has resulted in great interest and funding for development of sustainable energy sources.[1] One tapped source of sustainable energy involves conversion of lignocellulosic biomass into platform chemicals for conversion into bioenergy, biomaterials, or biochemicals.[2] This resource's viability is based on the fact that lignocellulosic biomass is predominantly comprised carbohydrates of cellulose and hemicellulose, which can be depolymerized into monosaccharide platform chemicals.[3-5]

Among the numerous lignocellulosic biomass candidates for conversion, bamboo is almost universally deemed viable because of its extraordinary growth rate, present availability, and high carbohydrate content (cellulose, 35-50\% and hemicellulose, 15-35\%).[6] To add to the case for bamboo, the vast majority of industrial bamboo residual waste materials are either landfilled or incinerated, serving as an untapped feedstock that is already within the supply chain. The greatest obstacle toward 
conversion of residual bamboo is a chemical one- its lignin is particularly recalcitrant to the biorefinery processing schemes currently demonstrated upon other biomass resources.[7] Thus, it is imperative to develop tailored pretreatment techniques capable of overcoming bamboo lignin's recalcitrance and allow for efficient production of platform monosaccharides.[8] Many different kinds of pretreatments have been studied upon bamboo substrates to date.[9] Among these pretreatment methods, dilute acid pretreatment appears to be most logical because it has been successfully applied pretreatment to bamboo residues, with the added benefit of low process costs and ease of operation.[10] However, the enzymatic digestibility of bamboo residues could not reached the expected extent and usually lower than $40 \%$.[11] The explanations for the low enzymatic saccharification efficiency have been proposed: 1) lignin-like compounds (pseudo-lignin) is be formed and problematically deposited on the surface of fibers.[12-13] 2) lignin undergoes repolymerization reactions which also block available surface area, and 3) water-soluble phenolics produced by depolymerization of lignin serve as soluble enzyme inhibitors.[14] However, the reported work mainly focus on the macroscopical effects of these lignin on enzymatic hydrolysis of pretreated biomass, the microcosmic effects of these lignin on cellulase adsorption that can influence the enzymatic hydrolysis is still scarce.

The interaction between lignin and cellulase is one of the important factors influencing enzymatic digestibility of pretreated biomass.[15] Techniques to study this 
interaction have been extensively explored. For instance, Langmuir adsorption isotherm was used to characterize enzyme affinity to lignin from the macro-scale.[16] Sodium dodecyl sulfate-polyacrylamide gel electrophoresis (SDS-PAGE) was applied to investigate cellulase-lignin affinity, but the results obtained were not quantitative.[17] In recent years, Quartz crystal microbalance with dissipation (QCM-D) has been relied upon to investigate the kinetic adsorption behavior in real time. [18-19] Surface plasma resonance (SPR), a novel method for measuring interactions between various compounds, has also been applied.[20] Like QCM technology, SPR also achieves real-time monitoring and analysis of interactions between a molecule and solids, allowing for acquisition of highly detailed information such as the kinetics of association and dissociation.[21-22] To our knowledge, there is no current work where SPR technology was applied to investigate the interactions between lignin and cellulase over the course of an enzymatic hydrolysis process.

In order to be able to evaluate the interactions between lignin and cellulase, a representative lignin preparation must be extracted from pretreated biomass (bamboo residues in this case). There are many different methods for extract lignin from pretreated-materials, but most are based around extraction using organic solvent such as 1,4-dioxane, tetrahydrofuran, ethanol, acetone, and more.[23-24] Hansen solubility parameter (HSP) theory typically drives the decisions for lignin solvent selection, taking into consideration the inter-molecular forces between solvents and lignin (dispersion, 
polarity and hydrogen bonding).[25] According to HSP theory, solvents with different solubility parameter have the ability to extract different lignin fractions from the same pretreated biomass substrate. Importantly, the lignin on the surface and cell wall of acid pretreated biomass, which is mostly likely to be engaging with cellulolytic enzymes, is quite homogeneous from different extractions. However, this material would certainly be the ideal lignin to serve as substrate for SPR analysis. Therefore, a variety of different solvents have been applied in this work to extract different lignin fractions in/on pretreated bamboo residues, in effort to evaluate the range of interactions that SPR can detect.

In this work, three different organic solvents (1,4-dioxane, ethanol, and tetrahydrofuran) with different HSP were used to extract different surface lignin fractions on DAP-BR. A classical lignin preparation (milled wood lignin, "MWL") was also extracted from the extracted residual solids, referred to as E-DAP-BR. Following extraction, enzymatic hydrolysis of DAP-BR, E-DAP-BR and mixtures of pure cellulose with different extracted lignin fractions were performed to evaluate the effects of different lignin fractions on enzymatic hydrolysis. The physicochemical properties of lignin (molecular weight and hydrophobicity) and DAP-BR (crystallinity indices, cellulose accessibility and hydrophobicity) were determined and used to probe for relationships with enzymatic digestibility. Furthermore, SPR technology was utilized to characterize the interaction between extracted lignin fractions and cellulase, with the 
kinetic constants accounting for their interactions being calculated. It is our hope that this work will provide a more detailed look at the potential mechanisms hindering digestion of pretreated bamboo by enzymes.

\section{Results and discussion}

\section{Influence of organic reagent extraction on chemical composition of dilute acid}

\section{pretreated bamboo residues}

Recently, Hansen solubility parameters (HSP) theory have become a popular tool for screening different organic solvent systems able to efficiently separate lignin from biomaterial matrices.[25] Based on HSP theory, if a given RED value $\left(R_{a} / R_{0}\right)$ is $<1$, the analyzed solvent will exhibit greater lignin affinity. Alternatively, when RED is $>1$, that means the chosen solvent system will behave as a poor lignin solvent.[26] Thus, in this work, three organic reagent systems (1,4-dioxane, ethanol, and tetrahydrofuran) were selected to extract different surface lignin fractions based on the difference in their RED values.

Table 1 Effects of three organic reagents extraction on chemical composition of dilute acid pretreated bamboo residues

\begin{tabular}{cccccccc}
\hline \multirow{2}{*}{ Biomass $^{\mathrm{a}}$} & \multicolumn{3}{c}{ Content(\%) } & \multicolumn{3}{c}{ Recovery yield(\%) } & \multicolumn{2}{c}{ Removal yield $(\%)$} \\
\cline { 2 - 7 } & Glucan & Xylan & Lignin & Solid & Glucan & Xylan & Lignin \\
\hline DAP-BR & $51.5 \pm 0.1$ & $2.7 \pm 0.0$ & $47.2 \pm 0.5$ & $/$ & $/$ & $/$ & $/$ \\
Dio-BR & $61.4 \pm 0.1$ & $3.0 \pm 0.1$ & $35.1 \pm 0.3$ & 82.8 & 98.5 & 1.3 & 38.5 \\
Eth-BR & $57.5 \pm 1.3$ & $2.8 \pm 0.1$ & $39.7 \pm 0.6$ & 91.3 & 100.0 & 1.1 & 23.2 \\
THF-BR & $60.1 \pm 0.1$ & $3.3 \pm 0.2$ & $38.3 \pm 0.5$ & 85.9 & 100.0 & 2.9 & 30.3 \\
\hline
\end{tabular}

a: DAP-BR:dilute acid pretreated bamboo residues. Dio-BR, Eth-BR, THF-BR: extracted by 1,4-dioxane extraction using concentration of $96 \%$ (v/v, water/1,4-dioxane), ethanol extraction using concentration of $95 \%$ (v/v, water/ethanal) and etrahydrofuran extraction using concentration 
of $100 \%(\mathrm{v} / \mathrm{v})$

The chemical compositions of extracted materials were determined and shown in

Table 1. Table 1 shows that the glucan and xylan fractions remained in DAP-BR after lignin extraction processes, meaning tested system was indeed lignin-selective.

According to Table 1, solids recovery of DAP-BR after 1,4-dioxane (Dio-BR), ethanol (Eth-BR), and tetrahydrofuran (THF-BR) was $82.8 \%, 91.3 \%$, and $85.9 \%$, respectively.

Based on lignin contents, 1,4-dioxane showed the best ability for lignin extraction (38.5\%), while ethanol performed the worst (23.2\%). The disparity between two solvent systems was consistent with their RED values of 0.91 and 1.37 , respectively. The difference in lignin extraction yields could also be confirmed by the SEM images of extracted DAP-BR, where lignin droplets were not observed on surfaces of extracted DAP-BR. In addition, comparing with different extracted DAP-BR, the surface of Dio-BR is smoother than that of Eth-BR.

\section{Influence of organic extraction agent on enzymatic digestibility and}

\section{physicochemical properties of pretreated bamboo.}

To evaluate the effect of removal of different surface lignin fractions on the enzymatic digestibility of DAP-BR, enzymatic hydrolysis was performed on both DAP-BR and extracted DAP-BR. Fig. 1a shows that the enzymatic hydrolysis yield of extracted DAP-BR significantly decreased from $36.5 \%$ (DAP-BR) to $18.6 \%$ (Dio-BR), $29.7 \%$ (THF-BR) and 30.2\% (Eth-BR). These results indicated that the removal of surface lignin actually decreased enzymatic digestibility. This finding was in alignment 
with the work of Lai et al (2019),[27] who found that that the enzymatic digestibility of organosolv acid-pretreated sawdust with 25\% (v/v) ethanol solution and 50\% (v/v) ethanol could decreased from $43.6 \%$ to $36.9 \%$ and from $50.0 \%$ to $42.5 \%$ when the surface lignin on pretreated substrate was removed by ethanol. Intuitively, the removed surface lignin on pretreated biomass would be thought to promote its enzymatic digestibility. However, the contrary results in this work comparing to the reported work might be due to the different solubility of certain lignin fractions in different organic solvents.[12,28-29] It is speculated that extraction of different surface lignin fractions with different physicochemical properties, which might promote the enzymatic digestibility of cellulose by enzyme. 
Table 2 Physicochemical properties of solvent extracted bamboo residues and extracted lignin fractions

\begin{tabular}{|c|c|c|c|c|c|c|c|c|}
\hline \multirow{2}{*}{ Sample $^{a}$} & \multirow{2}{*}{$\begin{array}{l}\text { Accessibility } \\
(\mathrm{mg} / \mathrm{g})\end{array}$} & \multirow{2}{*}{$\begin{array}{l}\text { Hydrophobicity } \\
(\mathrm{mg} / \mathrm{mL})^{\mathrm{b}} \text { and }\left(^{\circ}\right)^{\mathrm{c}}\end{array}$} & \multirow{2}{*}{$\begin{array}{l}\text { Crystallinity } \\
\qquad \%)\end{array}$} & \multirow{2}{*}{$\begin{array}{l}\mathrm{B}_{\mathrm{hkl}} \\
(\mathrm{nm})\end{array}$} & \multirow{2}{*}{$\begin{array}{l}\mathrm{D}_{\mathrm{hkl}} \\
(\mathrm{nm})\end{array}$} & \multirow{2}{*}{$\begin{array}{l}\text { Zeta Potential } \\
\qquad(\mathrm{mV})\end{array}$} & \multicolumn{2}{|c|}{ Molecular weight } \\
\hline & & & & & & & $\mathrm{M}_{\mathrm{w}}$ & $\mathrm{M}_{\mathrm{n}}$ \\
\hline DAP-BR & 218 & $0.7^{\mathrm{b}}$ & 38 & 3.03 & 0.403 & $-24.3 \pm 0.5$ & I & \\
\hline Dio-BR & 320 & $3.6^{b}$ & 36 & 3.64 & 0.401 & $-20.4 \pm 0.2$ & l & \\
\hline Eth-BR & 236 & $3.1^{b}$ & 39 & 3.45 & 0.402 & $-22.1 \pm 1.1$ & l & \\
\hline THF-BR & 251 & $3.0^{\mathrm{b}}$ & 34 & 3.99 & 0.400 & $-21.5 \pm 0.3$ & l & \\
\hline DAP-MWL & I & $76.4^{c}$ & I & I & I & $-37.0 \pm 0.3$ & 9140 & 2930 \\
\hline Dio-MWL & I & $76.2^{c}$ & I & I & l & $-31.8 \pm 0.2$ & 10810 & 3610 \\
\hline Eth-MWL & / & $73.8^{c}$ & l & / & l & $-34.3 \pm 0.3$ & 12990 & 4560 \\
\hline THF-MWL & / & $75.1^{\mathrm{c}}$ & I & / & I & $-37.0 \pm 0.3$ & 13200 & 4700 \\
\hline Dio-SL & I & $69.5^{c}$ & I & I & I & $-32.8 \pm 0.2$ & 2440 & 710 \\
\hline Eth-SL & / & $68.5^{c}$ & I & I & I & $-29.1 \pm 1.1$ & 1190 & 390 \\
\hline THF-SL & I & $64.3^{c}$ & I & I & I & $-26.1 \pm 0.1$ & 1980 & 390 \\
\hline DAP-MWL & / & $76.4^{c}$ & I & / & I & $-37.0 \pm 0.3$ & 9140 & 470 \\
\hline
\end{tabular}

a: DAP-BR:dilute acid pretreated bamboo residues. Dio-BR, Eth-BR, THF-BR: extracted by 1,4-dioxane extraction using concentration of $96 \%$ ( $\mathrm{v} / \mathrm{v}$, water/1,4-dioxane), ethanol extraction using concentration of $95 \%$ (v/v, water/ethanal) and etrahydrofuran extraction using concentration of $100 \%(\mathrm{v} / \mathrm{v})$; MWL: milled wood lignin, SL: surface lignin.

b: Analyzed by dye absorption

c: Analyzed by water contact angle 
To further investigate the reason why enzymatic digestibility of DAP-BR was decreased after being extracted using organic solvents, several physicochemical properties of DAP-BR and E-DAP-BR were evaluated to probe for stark changes (Table 2). It can be seen that the cellulose accessibility of DAP-BR increased from 218 $\mathrm{mg} / \mathrm{g}$ to $236 \mathrm{mg} / \mathrm{g}, 236 \mathrm{mg} / \mathrm{g}$ and $251 \mathrm{mg} / \mathrm{g}$ for Dio-BR, Eth-BR, and THF-BR, respectively. A slightly correlated relationship $\left(\mathrm{R}^{2}=0.68\right)$ between delignification and cellulose accessibility was observed (Fig. 1b). The increase noted for cellulose accessibility for cellulases is likely owed to the removal of lignin covered on the surface of cellulose. Generally, the improvement of cellulose accessibility tended to be the crucial factor to improve its enzymatic digestibility.[30] However, in this work, our results seem to indicate that cellulose accessibility was not an important factor for enzymatic hydrolysis under these specific details of sample workup. One possible hypothesis may be that after removing the surface lignin, more residual lignin is exposed on substrate's surface, foster a greater frequency of non-productive adsorption with cellulases. In support of this hypothesis, hydrophobicity analysis results showed that solvent extracted samples (Dio-BR, Eth-BR and THF-BR) had higher hydrophobicity $(3.6,3.1$, and $3.0 \mathrm{mg} / \mathrm{mL}$, respectively) compared to DAP-BR $(0.7 \mathrm{mg} / \mathrm{mL})$. This speculation is also supported by the linear fitting shown in Fig. 1c, where a negative correlation $\left(\mathrm{R}^{2}=0.68\right)$ can be found for the enzymatic hydrolysis yield of extracted DAP-BR and their hydrophobicity. Huang et al, 2019 also reported that substrates with higher hydrophobicity values induce more of non-productive 
binding by cellulase, reducing enzymatic hydrolysis yield.[31]

Cellulase is mainly consisted of Cellobiohydrolase I (CBH I or Cel7A), which is negatively charged during enzymatic hydrolysis. So, the higher negative zeta potential of lignin brought stronger electrostatic repulsion between lignin and substrates and reduced their non-productive binding. To further probe into our findings, zeta potentials of all samples were also analyzed (Table 2). It can be seen that all extracted DAP-BR have a much lower zeta potential (absolute value) than DAP-BR. Higher zeta potential (absolute value) values for substrates represents higher surface charge and more hydrophilic substrates.[32-33] Thus, more hydrophilic substrates also undergo stronger electrostatic repulsion between lignin and cellulase, reducing non-productive binding between lignin and cellulase to improve enzymatic hydrolysis. As shown in Fig. 2d, a negative correlation $\left(\mathrm{R}^{2}=0.83\right)$ was linearly fitted between enzymatic hydrolysis yields for each sample and their respective zeta potential. Results in Table 2 also show that there were no obvious differences for crystallinity index (CrI) and crystallite size (Bhkl, $\mathrm{D}_{\mathrm{hkl}}$ ) of cellulose for extracted DAP-BR and DAP-BR. These results illustrate that organic solvent extraction did not damage or change the cellulose, therefore these factors do not affect the enzymatic digestibility.

Based on the aforementioned results, it seems that the main driver between the enzymatic digestibility decrease after organic solvent extraction was exposure of highly hydrophobic lignin on the surface. To further probe this effect, residual lignin in extracted DAP-BR samples were further isolated and characterized to understand if 
it may play a more critical role on inhibition of enzymatic hydrolysis.

\section{Characterization of lignin fractions}

It is widely agreed that some physicochemical properties of lignin, such as molecular weight, hydrophobicity and surface charge, exert varying influence on enzymatic hydrolysis of cellulose.[34] Hence, certain physicochemical properties (molecular weight, contact angel, and zeta potential) of isolated SL samples on DAP-BR and MWL sample in E-DAP-BR were evaluated and shown in Table 2.

From Table 2, it can be seen that the molecular weight of DAP-MWL, Dio-MWL, Eth-MWL and THF-MWL were 9140-13200 g/mol (M $\left.{ }_{\mathrm{w}}\right)$ and 2930-4700 g/mol $\left(\mathrm{M}_{\mathrm{n}}\right)$, which were higher than that of Dio-SL, Eth-SL and THF-SL $\left(M_{\mathrm{w}}, 1190-1980 \mathrm{~g} / \mathrm{mol}\right.$, Mn, 390-710 g/mol). This was because that surface lignin was mixture of newly formed pseudo lignin and the formed monomer products of lignin during acid pretreatment. However, the milled wood lignin was residual lignin hidden in DAP-BR cell walls after the organic solvents extraction, which represented native lignin's chemical structure in substrates. These results were in accordance to the work of $\mathrm{Hu}$ et al, (2014),[35] who reported the molecular weights of pseudo-lignin were much lower than residual lignin in acid-pretreated biomass.Furthermore, the molecular weights of different surface lignin fractions were slightly different (Dio-SL 2440 g/mol, Eth-SL $1190 \mathrm{~g} / \mathrm{mol}$, and THF-SL $1980 \mathrm{~g} / \mathrm{mol}$ ). This variation is likely governed by each solvent's particular Hansen solubility parameters. Next, hydrophobicity of SL and MWL samples, evaluated as water contact angle, are also listed in Table 2. Water 
contact angles for MWL $\left(73.8^{\circ}-76.4^{\circ}\right)$ were much higher than SL $\left(64.3^{\circ}-69.5^{\circ}\right)$, which means the hydrophobicity of residual lignin in extracted DAP-BR is higher than that of surface lignin fraction on the DAP-BR. The results demonstrate that the increase to residual lignin hydrophobicity after solvent exposure of residual lignin is also likely contributing to the diminished digestibility noted after extraction with organic solvent.

Huang et al, (2017) reported that the lignin with higher negative zeta potentials generate higher repulsion to enzymes, resulting in improved enzymatic digestibility via reduced non-productive binding.[36] Hence, the zeta potentials of different lignin samples (SL and MWL) were also measured to compare their different effects on enzymatic hydrolysis. Table 2 shows that the negative zeta potentials of SL samples was less than those of MWL samples. Combined with the results of enzymatic hydrolysis, it can be seen that although the zeta potential of MWL in E-DAP-BR was higher, it may still cause more non-productive binding between enzyme and lignin on the substrate surface. This also suggests that the hydrophobic interactions and hydrogen binding were more important than the electrostatic interactions between lignin and enzyme, speculation also previously noted by Sun et al, 2016.[37]

\section{Effects of surface lignin and milled wood lignin on enzymatic hydrolysis of Avicel}

In order to further investigate the different effects of surface lignin and the residual lignin fractions on enzymatic digestibility of cellulose, all MWL and SL samples were dosed into enzymatic hydrolysis systems of Avicel (pure cellulose). Resultant enzymatic digestibility are shown in Fig. 2. It is observed that three SL fractions did 
not inhibit the enzymatic hydrolysis at $20 \%$ addition (Fig. 2a). Unexpectedly, a slight increase to enzymatic hydrolysis yield for Avicel was actually achieved, from $77.0 \%$ (control) to $81.2 \%$ (Dio-SL), $79.7 \%$ (Eth-SL), and 77.1\% (THF-SL) at $40 \%$ lignin addition (Fig. 2b). This phenomenon was similarly reported in the work of Wu et al, 2017.[38] In contrast, the enzymatic hydrolysis yield of Avicel were decreased from $77.0 \%$ (control) to $58.4 \%, 39.7 \%, 45.3 \%$, and $39.0 \%$ with $40 \%$ addition of DAP-MWL, Dio-MWL, Eth-MWL and THF-MWL (respectively) (Fig. 2c). These findings show that the residual lignin in extracted DAP-BR in indeed much more inhibitory than the SL fractions on DAP-BR, which is in agreement with aforementioned results. In addition, it is also found that the inhibition effect of DAP-MWL was relatively weaker than the MWL samples from extracted DAP-BR. This different might be because DAP-MWL was obtained from the DAP-BR, which also contained the surface lignin fractions. When surface lignin was removed from DAP-BR, the obtained Dio-MWL, Eth-MWL and THF-MWL were primarily comprised of lignin that can inhibit the enzymes during enzymatic hydrolysis process. Hence, it can be speculated that the presence of surface lignin on DAP-BR can retard residual lignin's potent inhibitory properties toward enzymatic digestibility

To understand if the reduced enzymatic digestibility of Avicel was related to any specific physicochemical properties of lignin samples, both the hydrophobicity and molecular weight of all obtained lignin were fitted to the enzymatic hydrolysis yield. It can be seen that a negative correlations with $\mathrm{R}^{2}$ of 0.68 (Fig. 2d) and 0.94 (Fig. 2e) were 
linearly fitted between water contact angle and molecular weight and enzymatic hydrolysis yield of Avicel, respectively. Similarly, Huang et al, (2017) added different lignin fractions to Avicel enzymatic hydrolysis and found similar correlations.[36]

As for the effects of lignin molecular weight, Zhao et al, (2020) recently reported that lignin with greater molecular weights have more non-productive adsorption with enzymes compared to those of lower molecular weights.[39] According to table 2, The Mw of SL (1185-1980 g/mol) was lower than these of MWL (9135-13200 g/mol), which indicated that SL have less non-productive adsorption with enzymes than MWL. Hence, it can be speculated that the residual lignin in DAP-BR and E-DAP-BR had higher hydrophobicity and molecular weight might showed more non-productive absorption between lignin and cellulase by enhancing the hydrophobic interactions, which can be attributed to their inhibitions on enzymatic hydrolysis yield of Avicel.

To further investigate the how cellulases may be adsorbing to the different lignin fractions investigated in this work, Chrastil's approach (Eq. 1) was used to study the diffusion-limited kinetic model of enzymatic hydrolysis system of Avicel doped with our lignin fractions.[40] The equation is as follows:

$$
P=P_{\delta}\left[1-\operatorname{EXP}\left(-K E_{o} t\right)\right]^{\wedge} n
$$

where $\mathrm{P}(\mathrm{g} / \mathrm{L})$ and $\mathrm{P} \sigma(\mathrm{g} / \mathrm{L})$ are the products which diffuse at every considered time $\mathrm{t}$ and at equilibrium, respectively. A rate constant, $\mathrm{k}\left(\mathrm{g} \cdot \mathrm{L}^{-1} \mathrm{~h}^{-1}\right)$ proportional to the diffusion coefficient as is defined by Fick's law.[41] $\mathrm{E}_{0}(\mathrm{~g} / \mathrm{L})$ is the initial enzyme concentration, and $\mathrm{n}$ is the structural diffusion resistance constant. The resistance 
constant is dependent on the steric structures of studied system. Generally, the more negative effects takes place in enzymatic hydrolysis system, the constant $\mathrm{n}$ is lower. Meanwhile, a lower $\mathrm{k}$ indicates that the substrate is more resistant to enzymatic hydrolysis, translating to decreased engagement between the enzyme and substrate. Table 3 Dynamics parameters of the determined Chrastil model from the experimental results.

\begin{tabular}{cccc}
\hline Sample & $\begin{array}{c}\mathrm{k}^{\mathrm{a}} \\
\left(\mathrm{g} . \mathrm{L}^{-1} \mathrm{~h}^{-1}\right)\end{array}$ & $\mathrm{n}^{\mathrm{b}}$ & $\mathrm{R}^{2}$ \\
\hline Avicel & $6.25 \mathrm{E}-03$ & 0.51 & 0.9911 \\
Avicel+DAP-MWL & $9.10 \mathrm{E}-05$ & 0.43 & 0.9965 \\
Avicel+Dio-MWL & $9.75 \mathrm{E}-05$ & 0.39 & 0.9926 \\
Avicel+ Eth-MWL & $8.60 \mathrm{E}-05$ & 0.44 & 0.9985 \\
Avicel+THF-MWL & $9.94 \mathrm{E}-05$ & 0.42 & 0.9866 \\
Avicel+ Dio-SL & $2.27 \mathrm{E}-04$ & 0.50 & 0.9858 \\
Avicel+ Eth-SL & $1.10 \mathrm{E}-03$ & 0.50 & 0.9985 \\
Avicel+THF-SL & $6.25 \mathrm{E}-03$ & 0.54 & 0.9975 \\
\hline
\end{tabular}

a: $k$, a rate constant defined by Fick's law, proportional to the diffusion coefficient.

$\mathrm{b}: \mathrm{n}$, structural diffusion resistance constant.

In this work, the model parameters for enzymatic hydrolysis of Avicel-lignin system were determined and shown in Table 3. For all systems, good agreement with the experimental results was obtained $\left(\mathrm{R}^{2}>0.98\right)$, indicating the kinetic models for enzymatic hydrolysis of the Avicel-lignin systems were significant. According to the obtained results, $\mathrm{n}$ value of Avicel digestion decreased from 0.51 to $0.43,0.39,0.44$, and 0.42 when DAP-MWL, Dio-MWL, Eth-MWL, and THF-MWL were dosed into enzymatic hydrolysis process, respectively. Contrarily, the addition of any of three SL samples with Avicel did not affect n value. This indicates that the extracted surface 
lignin had little-to-no effect on the diffusion resistance of the enzyme and substrate resistance during enzymatic hydrolysis. Hence, it can be speculated that with the addition of MWL samples, the cellulase was subjected a stronger diffusion resistance to Avicel. This is attributable to the decreased enzymatic hydrolysis yield. Also, $\mathrm{k}$ values also decreased upon addition of MWL to the hydrolysis system, while the addition of SL had little effect on its k values. These results again demonstrated that residual lignin in extracted DAP-BR showed a higher diffusion resistance of the enzyme and substrate resistance to enzymatic hydrolysis. This is in agreement with the results of enzymatic digestibility in Fig.2. To verify our conjecture, the interaction mechanisms between enzyme and each different fraction was further investigated at the molecular level.

\section{Enzyme-lignin surface interaction determined by SPR technology}

SPR technology has been widely applied to study the adsorption between proteins and biomacromolecules. To evaluate the interaction and affinity between enzymes (protein) and lignin fractions (biomacromolecules), SPR was used to real-time monitor the association and dissociation process of cellulase enzymes on generated lignin. The dynamic processes of different SL and MWL samples for cellulase binding in real time is shown in Fig. 3. From 0 to $200 \mathrm{~s}$, The citric acid buffer was injected to obtain a flat baseline. Then from 200 to 440 s, different concentrations of cellulase solution $(0.1$, $0.05,0.02,0.01$, and $0.005 \mathrm{~g} / \mathrm{L}$ ) were injected to adsorb with lignin, resulting in changes of RU (SPR Response Unit, $1 \mathrm{RU}=1 \times 10^{-6} \mathrm{RIU}, 1 \mu \mathrm{RIU}=0.15 \mathrm{mdeg}$ ) values. 
After 440s, citric acid buffer was injected again, lignin and cellulase were disintegrated, resulting in the decrease of RU value. Specific enzyme kinetics adsorption parameters on lignin films were calculated and shown in Table 4. It can be observed that the values of RU increased when enzyme bonded to the lignin and decreased when the enzyme dissociated from lignin after addition of buffer solution into the instrument. These observations showed that the decrease of RU in SL-cellulase systems were more obvious than these in MWL-cellulase system during enzyme dissociation process, indicating the enzymes were much more easily dissociated from SL compared MWL.

Table 4 Enzyme kinetics adsorption parameters on lignin films as measured by SPR.

\begin{tabular}{ccccc}
\hline Lignin & $\begin{array}{c}\mathrm{k}_{\mathrm{a}}^{\mathrm{a}} \\
\left(\mathrm{M}^{-1} \mathrm{~S}^{-1}\right)\end{array}$ & $\begin{array}{c}\mathrm{k}_{\mathrm{d}}^{\mathrm{b}} \\
\left(\mathrm{S}^{-1}\right)\end{array}$ & $\begin{array}{c}\mathrm{R}_{\max }{ }^{\mathrm{c}} \\
(\mathrm{RU})\end{array}$ & $\begin{array}{c}\mathrm{K}_{\mathrm{D}}{ }^{\mathrm{d}} \\
(\mathrm{nM})\end{array}$ \\
\hline DAP-MWL & $3.1 \times 10^{4}$ & $7.68 \times 10^{-4}$ & 497 & 24.7 \\
Dio-MWL & $4.0 \times 10^{4}$ & $2.70 \times 10^{-4}$ & 433 & 6.8 \\
Eth-MWL & $3.1 \times 10^{4}$ & $5.10 \times 10^{-4}$ & 406 & 16.3 \\
THF-MWL & $3.3 \times 10^{4}$ & $4.28 \times 10^{-4}$ & 411 & 13.2 \\
Dio-SL & $3.4 \times 10^{4}$ & $1.57 \times 10^{-3}$ & 493 & 48.9 \\
Eth-SL & $2.7 \times 10^{4}$ & $1.46 \times 10^{-3}$ & 506 & 52.6 \\
THF-SL & $5.5 \times 10^{4}$ & $2.18 \times 10^{-3}$ & 486 & 39.4 \\
\hline
\end{tabular}

$\mathrm{a}: \mathrm{k}_{\mathrm{a}}$, association rate constant

$\mathrm{b}: \mathrm{k}_{\mathrm{d}}$, dissociation rate constant of $\mathrm{k}_{\mathrm{d}}$

c: $\mathrm{R}_{\max }$, the maximum amount of cellulase bound to lignin film

d: $K_{D}$, equilibrium dissociation constant

Increased $\mathrm{k}_{d}$ and $\mathrm{R}_{\max }$ values in Table 4 indicate that cellulase was more associated and faster dissociated from the SL samples than MWL samples. $K_{D}$, an indicator for binding affinity of enzymes and lignin, was different between the systems with SL 
versus MWL. Specifically, the affinity $\left(\mathrm{K}_{\mathrm{D}}\right)$ was $6.8-24.7 \mathrm{nM}$ for enzyme and MWL versus 39.4-52.6 $\mathrm{nM}$ for enzyme and SL. Generally, lower values for $\mathrm{K}_{\mathrm{D}}$ represent the stronger affinity of substrate for the adsorbate. Hence, it can be concluded that the removal of the SL fractions from DAP-BR via solvent extraction did likely expose a new layer of residual lignin that allowed for more non-productive binding and an overall decrease in enzymatic digestion. This speculation can be verified by linearly fitting $K_{D}$ values and their enzymatic digestibility, where a negative correlation $\left(R^{2}=0.91\right.$, Fig. $\left.4 a\right)$ and a positive correlation $\left(R^{2}=0.47\right.$, Fig. $\left.4 b\right)$ were observed for MWL-cellulase and SL-cellulase systems. In addition, an excellent negative correlation $\left(\mathrm{R}^{2}=0.95\right.$, Fig. $\left.4 \mathrm{c}\right)$ was fitted between enzymatic hydrolysis of Avicel and the $\mathrm{K}_{\mathrm{D}}$ values of SL samples (higher values) and MWL samples (lower values). These differences indicate that the SL fractions on DAP-BR had lower affinity for adsorption to cellulose.

To understand if the differences in affinity constant between lignin and enzyme was related to the physicochemical properties of lignin, the hydrophobicity and molecular weight of all obtained lignin fractions were fitted to their affinity values. Fig. $4 \mathrm{~d}$ showed a positive correlation $\left(\mathrm{R}^{2}=0.88\right)$ was linearly fitted between lignin molecular weight and affinity values for enzyme and lignin. In addition, hydrophobicity of SL and MWL samples were linearly fitted $\left(\mathrm{R}^{2}=0.68\right.$, Fig. $\left.4 \mathrm{e}\right)$ with affinity values between lignins and enzyme. Hence, it can be concluded that hydrophobic interaction between lignin and cellulase are the kinds of affinity behavior 
during enzymatic hydrolysis, playing a negative role for enzymatic conversion.

Similar conclusions are also drawn in the work of Li et al, (2016).[42] Overall, these SPR results indicate that the method can be used as a good technology to investigate the interaction mechanism between lignin and enzymes.

\section{Conclusions}

In this work, removal of different surface lignin fractions on DAP-BR showed a reduction effect on the resultant solid's enzymatic digestibility due to the exposure of residual lignin with higher hydrophobicity and molecular weight. The residual lignins in extracted DAP-BR had higher diffusion resistance to enzymes in a cellulose-lignin system, while SL fractions did not show any substrate resistance. SPR results showed that residual lignin in E-DAP-BR with greater hydrophobicity had higher affinity with cellulase than that of SL on DAP-BR, which is linearly correlated to their negative effects on enzymatic hydrolysis.

\section{Materials and methods}

\section{Materials}

Bamboo residues were sampled from a bamboo processing factory in Sichuan,

China. The chemical contents of glucan, xylan, and lignin in bamboo residues were $40.1 \%, 22.0 \%$, and 27.2\%, respectively. Cellulase (Cellic CTec2) was provided by Novozymes NA, Franklinton, USA with a filter paper activity of 250.0 FPU/ml.

\section{Dilute acid pretreatment for bamboo residues}

For pretreatment, $1.0 \mathrm{~kg}$ bamboo residues were massed and then mixed with $6.0 \mathrm{~L}$ 
of $1 \%(\mathrm{w} / \mathrm{v})$ dilute sulfuric acid inside of a $15 \mathrm{~L}$ reactor at $160{ }^{\circ} \mathrm{C}$ for $1 \mathrm{~h}$. After pretreatment, the resultant liquor (prehydrolyzate) was collected via vacuum filtration. Captured solids (DAP-BR) were washed using distilled water until the wash filtrate $\mathrm{pH}$ was 7.0. Some of the washed DAP-BR was kept at $4{ }^{\circ} \mathrm{C}$ for enzymatic hydrolysis, while another portion was air-dried before further lignin extraction.

\section{Organic extraction for surface lignin from dilute acid pretreated bamboo residues}

Air-dried DAP-BR was respectively extracted by a 1,4-dioxane extraction/water solution $(96: 4, \mathrm{v} / \mathrm{v})$, a $95 \%$ ethanol aqueous solution, and a tetrahydrofuran (100\%) to prepare three unique surface lignin preparations. All extraction processes were carried out in 2.0 L conical flasks with $10 \%$ solids suspension at $150 \mathrm{rpm}$ for $24 \mathrm{~h}$. After $24 \mathrm{~h}$, the liquor was separated from residual solids by filter paper and fresh organic solution was again added to once-extracted solids. This process was totally repeated for three times. All of the extract liquids were combined and then subjected to evaporation via a rotary evaporator and a vacuum freeze dryer to obtain solids. Lignin solid preparations from solutions of 96\% 1,4-dioxane extraction/water, 95\% ethanol, and tetrahydrofuran were termed as Dio-SL, Eth-SL and THF-SL, respectively.

\section{Extraction of milled wood lignin from DAP-BR and E-DAP-BR}

The un-extracted/residual lignin in DAP-BR, Dio-BR, Eth-BR and THF-BR were next obtained using the milled wood lignin (MWL) preparation technique according to Bjorkman, (1954).[43] Specifically, $5.0 \mathrm{~g}$ (oven dry) of solids were weighed into a planetary ball mill (Pulverisette 7, Fritsch, Germany) and subjected to $6 \mathrm{~h}$ milling time 
at $600 \mathrm{rpm}$ using 25 zirconia balls with diameter of $1.0 \mathrm{~cm}$. The detail extraction and purification for the MWL preparation were according to our previous work.[44] Extracted MWL samples from DAP-BR, Dio-BR, Eth-BR and THF-BR solids are termed as DAP-MWL, Dio-MWL, Eth-MWL and THF-MWL, respectively.

\section{Enzymatic hydrolysis}

The enzymatic hydrolysis of preparation DAP-BR and E-DAP-BR were carried out at a substrate loading of $5 \%(\mathrm{w} / \mathrm{v})$ in $0.05 \mathrm{M}$ sodium citrate $(\mathrm{pH} 4.8)$ buffer with 20 FPU/g cellulase. Enzymatic hydrolysis was performed in $100-\mathrm{mL}$ flasks at $50{ }^{\circ} \mathrm{C}$ and $150 \mathrm{rpm} / \mathrm{min}$ for $72 \mathrm{~h}$. Hydrolysate aliquots were withdrawn from the hydrolysis suspension at certain time intervals and then centrifuged at 10,000 rpm for $5 \mathrm{~min}$. The enzymatic hydrolysis of a mixture substrate of Avicel with different additions of lignin $(20 \%, 40 \%, \mathrm{w} / \mathrm{w})$ was consistent with the above reaction conditions. The concentrations of glucose in hydrolysate aliquot was determined using an Agilent 1200 high-performance liquid chromatography (HPLC) system. Enzymatic digestibility of substrate was calculated as the following equation:

Enzymatic hydrolysis digestibility $(\%)=\frac{\text { glucose in enzymatic hydrolyzate }(\mathrm{g})}{\text { initial glucan in substrate }(\mathrm{g}) \times 1.11} \times 100 \%$

\section{Characterization of DAP-BR and E-DAP-BR}

Morphological images of dilute acid pretreated and organosolv extracted bamboo residues were taken using a Hitachi SEM (Scanning electron microscope, 3400-I, Hitachi, Japan) with an accelerating voltage of $15 \mathrm{kV}$.

The accessibility of DAP-BR and E-DAP-BR were analyzed by Congo red (Direct 
Red 28) adsorption assay according to the work of Inglesby and Zeronian, (2002).[45]

Adsorption of congo red was carried out in $50 \mathrm{ml}$ conical flasks with a substrate loading of $1 \%$ with different concentration of Congo $(0,0.05,0.1,0.5,1.0,2.0,3.0$ and $4.0 \mathrm{~g} / \mathrm{L})$.

Each sample was incubated in a constant temperature shaker $\left(60^{\circ} \mathrm{C}, 150 \mathrm{rpm}\right)$ for $24 \mathrm{~h}$. The Langmuir adsorption isotherm was used to fit the extent of adsorbed dye onto each substrate. These results were then interpreted as an indication of the cellulose accessibility for the applied cellulase cocktail.

The hydrophobicity of DAP-BR and E-DAP-BR were estimated using the Rose Bengal dye adsorption assay. A series of substrate concentrations $(2,4,6,8,10 \mathrm{~g} / \mathrm{L})$ were added into $50 \mathrm{mmol}$ citrate buffer $(\mathrm{pH} 4.8)$ at a constant concentration of 40 mg/L Rose Bengal solution. Mixed samples were kept inside a constant temperature shaker for $2 \mathrm{~h}\left(50{ }^{\circ} \mathrm{C}, 150 \mathrm{rpm}\right)$. The amount of adsorbed dye on each tested substrate was calculated by the difference between the initial dye in the solution and the absorbance after adsorption. A partition quotient (PQ) was then defined as the ratio of the amount of absorbed dye to the amount of residual free dye. Obtained PQ values were finally linearly fitted to the corresponding concentrations of samples, and the slope from each line of fit was used to represent surface hydrophobicity (L/g).

X-Ray diffraction (XRD) measurements for DAP-BR and E-DAP-BR were conducted by an Ultima IV diffractometer (Rigaku Corporation) at a voltage of $40 \mathrm{kV}$ and a current of $40 \mathrm{~mA}$. Scan speed was $10^{\circ} / \mathrm{min}$ at range of $5^{\circ}-50^{\circ}$. Both crystallinity index $(\mathrm{CrI})$ and crystallite size of cellulose $\left(\mathrm{B}_{\mathrm{hkl}}\right)$ were fitted using Mau Rietveld 
software (version 2.7) and calculated according to the equations from Ling et al. 2017.[46]

\section{Characterization of surface lignin and milled wood lignin}

Molecular weights of lignin samples were estimated by gel permeation chromatography (GPC) within a high-performance liquid chromatography (HPLC) system (Agilent 1200, Palo Alta, CA, USA) equipped with three Styragel columns (HR5E, HR4, and HR2) in tandem and refractive index detector. For analysis, $50 \mu \mathrm{L}$ of sample (4 mg/ml in tetrahydrofuran) was injected into the system. GPC calibration was performed according to commercial polystyrene standards.

Hydrophobicity analysis for all lignin samples was performed by water contact angle measurement using automatic contact angle meter (Attention Theta, Biolin Scienfic, Inc. Stockholm, Sweden). Each sample was tested in duplicate.

The zeta potentials of lignin samples were measured by a Zetasizer (Nano-ZS, Malvern Instruments Ltd, Worcestershire, UK) with laser Doppler. For analysis, $2.5 \mathrm{mg}$ of lignin was blended with $5 \mathrm{~mL}$ of $0.05 \mathrm{M}$ citrate buffer and dispersed using an disperser to get the homogeneous suspension. All samples were tested in triplicate.

\section{Enzyme-lignin surface interaction studied by SPR}

To prepare the lignin film needed as a SPR substrate, either SL or MWL samples were dissolved into neat DMSO at $0.5 \%$ solids loading $(\mathrm{w} / \mathrm{w})$. The prepared solutions were coated on a SPR gold sensor using a spin coater (KW-4A, Shanghai Daojing Instrument Plant, China) at $5000 \mathrm{rpm}$ for $1 \mathrm{~min}$. The coating process was repeated 3 
times. Resultant films were vacuum dried at $40{ }^{\circ} \mathrm{C}$ for $4 \mathrm{~h}$, and then soaked in the deionized water for $24 \mathrm{~h}$. The deionized soak water was replaced every $2 \mathrm{~h}$ to ensure complete removal of DMSO. The soaked films were vacuum dried at $40{ }^{\circ} \mathrm{C}$ for $12 \mathrm{~h}$. Interaction between lignin films and enzyme was measured by a SPR device (MP-SPR Navi 200, BioNavis Ltd, Tampere, Finland) equipped with a two channel detection system at $670 \mathrm{~nm}$ laser and angular-scan range of 40-78 degrees. Before injecting enzyme into the SPR system, 0.05 M citric acid buffer solution ( $\mathrm{pH} 4.8$ ) was injected into the measuring chamber at a flow rate of $100 \mathrm{~mL} / \mathrm{min}$ until a stable baseline was obtained. Once stable, the enzyme solutions with at protein concentrations $(0.1$, $0.05,0.02,0.01$, and $0.005 \mathrm{~g} / \mathrm{L}$ ) were injected at a flow rate of $0.1 \mathrm{~mL} / \mathrm{min}$ for 4 minutes. The recorded SPR curves were processed using SPR Navi ${ }^{\mathrm{TM}}$ Data Viewer software. Kinetics constants were determined using Scrubber (version 2.0) software.

\section{Analytical methods}

The composition of pretreated and organosolv-extracted bamboo residues were determined according to the procedure developed by the National Renewable Energy Laboratory (NREL).[47] Monosaccharide concentrations for enzymatic hydrolysate and compositional analysis acid hydrolysate were measured using a high performance liquid chromatography (HPLC) system equipped with an Aminex HPX-87H column $(300 \times 7.8 \mathrm{~mm})$ and a refractive index detector (column temperature of $55^{\circ} \mathrm{C}$, mobile phase of $0.05 \mathrm{M} \mathrm{H}_{2} \mathrm{SO}_{4}$ at a flow rate of $0.6 \mathrm{ml} / \mathrm{min}$ ). 
Recovery yields of solid or glucan, as well as the extent of delignification or xylan removal, were determined according to the following equations: Recovery yield of solid $(\%)=\frac{\text { pretreatedbamboo residue }(\mathrm{g})}{\text { initial bamboo residue }(\mathrm{g})} \times 100 \%$ Recovery yield of glucan $(\%)=\frac{\text { glucan in pretreated bamboo residue }(\mathrm{g})}{\text { glucan in the initial bamboo residue }(\mathrm{g})} \times 100 \%$ Removal yield of lignin or xylan $(\%)=1-\frac{\text { lignin or xylan in pretreatedbamboo residue }(\mathrm{g})}{\text { lignin or xylan in the initial bamboo residue }(\mathrm{g})} \times 100 \%$

\section{Abbreviations}

DAP-BR: dilute acid pretreated bamboo residues; SL: milled wood lignin; MWL: milled wood lignin;Dio-BR, Eth-BR,THF-BR: extracted by 1,4-dioxane extraction using concentration of $96 \%$ (v/v, water/1,4-dioxane), ethanol extraction using concentration of $95 \%(\mathrm{v} / \mathrm{v}$, water/ethanal) and etrahydrofuran extraction using concentration of $100 \%(\mathrm{v} / \mathrm{v})$; E-DAP-BR: dilute acid pretreated bamboo residues extracted by organic solvents; PQ: partition quotient; HPLC: high performance liquid chromatography; XRD: X-Ray diffraction; CrI: crystallinity index; Bhkl: crystallite size of cellulose; GPC:gel permeation chromatography; HSP: hansen solubility parameters; RED : relative energy difference; Mw: weight average molecular weight; Mn: number average molecular weight; k: a rate constant defined by Fick's law, proportional to the diffusion coefficient.; $\mathrm{n}$ : structural diffusion resistance constant; ka: association rate constant; $\mathrm{k}_{\mathrm{d}}$ : dissociation rate constant; $\mathrm{R}_{\max }$ : the maximum amount of cellulase bound to lignin film; $\mathrm{K}_{\mathrm{D}}$ : equilibrium dissociation constant

\section{Supplementary Information}


The online version contains supplementary material

Additional file 1: Table S1 RED and Hansen Solubility Parameters of 3 Solvents.

Additional file 2: Fig. S1 SEM images of pretreated bamboo residues (a), DAP-BR (b),

Dio-BR (c), Eth-BR and (d), THF-BR.

\section{Acknowledgements}

We thank Dr. Robert Narron in North Carolina State University to help us edit the languish of this manuscript. In addition, we thank Pro. Junlong Song in Nanjing Forestry University provide the SPR instrument for us.

\section{Authors' contributions}

WL and JY conceived the work and write the manuscript. YZ did the composition analysis of pretreated bamboo residues. $\mathrm{CH}$ revised the manuscript. QY provided the idea to do the SPR analysis. All authors read and approved the final manuscript.

\section{Funding}

This work was supported by Natural Science Foundation of Jiangsu Province (BK20180772), National Natural Science Foundation of China (31800501) and

Science and Technology Planned Projects of Zhejiang Province (2018F10009).

\section{Availability of data and material}

All data generated and analyzed in this study are included in this published article.

\section{Declarations}

\section{Ethics approval and consent to participate}

Not applicable. 


\section{Consent for publication}

The authors hereby consent to publication of this work in Biotechnology for Biofuels.

\section{Competing interests}

The authors declare that they have no competing interests.

\section{References}

1 Razmjoo A A, Sumper A, Davarpanah A. Development of sustainable energy indexes by the utilization of new indicators: A comparative study. Energy Rep. $2019 ; 5: 375-83$

2 Mahmood N, Wang Z, Yasmin N, Manzoor W, Rahman AU. How to bend down the environmental kuznets curve: the significance of biomass energy. Environ SCI PolluT R. 2019;26(21):21598-608.

3 Zhang J, Wang Y, Du X. Selective removal of lignin to enhance the process of preparing fermentable sugars and platform chemicals from lignocellulosic biomass. Bioresour Technol. 2020;303:122846.

4 Cao F, Xia S, Yang X. Lowering the pyrolysis temperature of lignocellulosic biomass by $\mathrm{H} 2 \mathrm{SO} 4$ loading for enhancing the production of platform chemicals. Chem Eng J. 2020;385:123809.

5 Dedes G, Karnaouri A, Topakas E. Novel routes in transformation of lignocellulosic biomass to furan platform chemicals: From pretreatment to enzyme catalysis. Catalysts. 2020;10(7):743. 
$6 \mathrm{Gu}$ T, Wang B, Zhang Z, et al. Sequential pretreatment of bamboo shoot shell and biosynthesis of ethyl (R)-4-chloro-3-hydroxybutanoate in aqueous-butyl acetate media. Process Biochem. 2019;80:112-8.

7 Zhang $\mathrm{X}, \mathrm{Xu} \mathrm{C}$, Wang $\mathrm{H}$. Pretreatment of bamboo residues with Coriolus versicolor for enzymatic hydrolysis. J Biosci Bioeng. 2007;104 (2):149-51.

8 Vieira S, Barros MV, Sydney ACN, Piekarski, CM, de Francisco AC, Sydney EB. Sustainability of sugarcane lignocellulosic biomass pretreatment for the production of bioethanol. Bioresour Technol. 2020;299:122635.

9 Yang G, Wang J. Ultrasound combined with dilute acid pretreatment of grass for improvement of fermentative hydrogen production. Bioresour Technol. $2019 ; 275: 10-8$

10 Leenakul W, Tippayawong N. Dilute acid pretreatment of bamboo for fermentable sugar production. J Sus Energ Environ. 2010;1(3):117-20.

11 Huang C, He J, Li X. Facilitating the enzymatic saccharification of pulped bamboo residues by degrading the remained xylan and lignin-carbohydrates complexes. Bioresour technol. 2015;192:471-7.

12 Hu F, Jung S, Ragauskas A. Pseudo-lignin formation and its impact on enzymatic hydrolysis. Bioresour Technol. 2012;117:7-12.

13 Schmatz AA, Salazar-Bry,am AM, Contiero J. Pseudo-Lignin Content Decreased with Hemicellulose and Lignin Removal, Improving Cellulose Accessibility, and Enzymatic Digestibility. BioEnergy Res. 2020;1-16. 
14 Lai C, Yang B, He J, Huang C, Li X, Song X, Yong Q. Enhanced enzymatic digestibility of mixed wood sawdust by lignin modification with naphthol derivatives during dilute acid pretreatment. Bioresour Technol. 2018;269:18-24.

15 Zhang L, Zhang L, Zhou T, Wu Y, Xu F. The dual effects of lignin content on enzymatic hydrolysis using film composed of cellulose and lignin as a structure model. Bioresour Technol. 2016;200:761-9.

16 Ying W, Shi Z, Yang H, Xu G, Zheng Z, Yang J. Effect of alkaline lignin modification on cellulase-lignin interactions and enzymatic saccharification yield. Biotechnol Biofuels. 2018;11(1):214.

17 Li M, Yi L, Bin L, et al. Comparison of nonproductive adsorption of cellulase onto lignin isolated from pretreated lignocellulose. Cellulose, 2020, 27(14): 7911-7927.

18 Kumagai A, Lee SH, Endo T. Evaluation of the effect of hot-compressed water treatment on enzymatic hydrolysis of lignocellulosic nanofibrils with different lignin content using a quartz crystal microbalance. Biotechnol Bioeng. 2016;113(7):1441-7.

19 Cui M, Duan Y, Ma Y, Al-Shwafy K W, Liu Y, Zhao X, Su R. Real-Time QCM-D Monitoring of the Adsorption-Desorption of Expansin on Lignin. Langmuir. 2020;36:4503-10.

20 Salehabadi H, Khajeh K, Dabirmanesh B, Biglar M, Amanlou M. Evaluation of angiotensin converting enzyme inhibitors by SPR biosensor and theoretical studies. Enzyme. Microb Tech. 2019;120:117-23. 
21 Chen X, Xu H, Wu N, Liu X, Qiao G, Su S, Lin X. Interaction between granulin A and enolase 1 attenuates the migration and invasion of human hepatoma cells. Oncotarget. 2017;8(18):30305.

22 Mohammadzadeh-Asl S, Aghanejad A, Yekta R, de la Guardia M, Dolatabadi JEN, Keshtkar A. Kinetic and thermodynamic insights into interaction of erlotinib with epidermal growth factor receptor: Surface plasmon resonance and molecular docking approaches. Int J Biol Macromol. 2020;163:954-8.

23 Yao L, Yang H, Yoo CG, Meng X, Pu Y, Hao N, Ragauskas AJ. Characteristics of lignin from dilute acid pretreated switchgrass and their effect on cellobiohydrolase from Trichoderma longibrachiatum. Front Energy Res. 2018;6:1-9.

24 Smith MD, Mostofian B, Cheng X, Petridis L, Cai CM, Wyman CE, Smith JC. Cosolvent pretreatment in cellulosic biofuel production: effect of tetrahydrofuran-water on lignin structure and dynamics. Green Chem. 2016;18(5):1268-77.

25 Zhang Q, Tan X, Wang W, Yu Q, Wang Q, Miao C, Yuan Z. Screening solvents based on Hansen solubility parameter theory to depolymerize lignocellulosic biomass efficiently under low temperature. ACS Sustain Chem Eng. 2019;7(9):8678-86.

26 Novo LP, Curvelo AA. Hansen solubility parameters: a tool for solvent selection for organosolv delignification. Ind Eng Chem Res. 2019;58(31):14520-7.

27 Lai C, Yang B, Lin Z, Jia Y, Huang C, Li X, Yong Q. New strategy to elucidate the 
positive effects of extractable lignin on enzymatic hydrolysis by quartz crystal microbalance with dissipation. Biotechnol Biofuels. 2019;12(1):1-12.

28 Sannigrahi P, Kim DH, Jung S, Ragauskas A. Pseudo-lignin and pretreatment chemistry. Energ Environ Sci. 2011;4(4):306-10.

29 Meng X, Ragauskas AJ. Pseudo-lignin formation during dilute acid pretreatment for cellulosic ethanol. Recent Adv Petrochem Sci. 2017;1(1).

30 Kumar L, Arantes V, Chandra R, Saddler J. The lignin present in steam pretreated softwood binds enzymes and limits cellulose accessibility. Bioresour Technol. 2012;103(1):201-208.

31 Huang C, Fang G, Yu L, Zhou Y, Ragauskas AJ. Maximizing enzymatic hydrolysis efficiency of bamboo with a mild ethanol-assistant alkaline peroxide pretreatment. Bioresour Technol. 2019;299:122568.41

32 Zheng W, Lan T, Li H, Zhou H. Exploring why sodium lignosulfonate influenced enzymatic hydrolysis efficiency of cellulose from the perspective of substrate-enzyme adsorption. Biotechnol Biofuels. 2020;13(1):19.

33 Xing Y, Bu L, Zheng T, Liu S, Jiang J. Enhancement of high-solids enzymatic hydrolysis of corncob residues by bisulfite pretreatment for biorefinery. Bioresour Technol. 2016;221:461-8.

34 Yang Q, Pan X. Correlation between lignin physicochemical properties and inhibition to enzymatic hydrolysis of cellulose. Biotechnol Bioeng. 2016;113(6):1213-24. 
$35 \mathrm{Hu} \mathrm{F}$, Ragauskas A. Suppression of pseudo-lignin formation under dilute acid pretreatment conditions. Rsc Adv. 2014;4(9):4317-23.

36 Huang Y, Sun S, Huang C, Yong Q, Elder T, Tu M. Stimulation and inhibition of enzymatic hydrolysis by organosolv lignins as determined by zeta potential and hydrophobicity. Biotechnol Biofuels. 2017;10(1):1-11.

37 Sun S, Huang Y, Sun R, Tu M. The strong association of condensed phenolic moieties in isolated lignins with their inhibition of enzymatic hydrolysis. Green Chem. 2016;18(15):4276-86.

$38 \mathrm{Wu}$ K, Shi Z, Yang H, Liao Z, Yang J. Effect of ethanol organosolv lignin from bamboo on enzymatic hydrolysis of avicel. ACS Sustain Chem Eng. 2017;5(2):1721-9.

39 Zhao C, Qiao X, Shao Q, Hassan M, Ma Z. Evolution of the Lignin Chemical Structure during the Bioethanol Production Process and Its Inhibition to Enzymatic Hydrolysis. Energy Fuel. 2020;34(5):5938-47.

40 Chrastil J. Enzymic product formation curves with the normal or diffusion limited reaction mechanism and in the presence of substrate receptors. Int $\mathrm{J}$ Biochem. 1988;20(7):683-93.

41 Crank J. The mathematics of diffusion. Oxford university press. 1979.

42 Li Y, Qi B, Luo J, Wan Y. Effect of alkali lignins with different molecular weights from alkali pretreated rice straw hydrolyzate on enzymatic hydrolysis. Bioresour Technol. 2016;200:272-8. 
43 Bjorkman, Anders. Isolation of lignin from fifinely divided wood with neutral solvents. Nature.1954;174:1057-8.

$44 \mathrm{He}$ J, Huang C, Lai C, Jin Y, Yong Q. Investigation of the effect of lignin/pseudo-lignin on enzymatic hydrolysis by Quartz Crystal Microbalance. Ind Crop Prod. 2020;157:112927.

45 Inglesby MK, Zeronian SH. Direct dyes as molecular sensors to characterize cellulose substrates. Cellulose. 2002;9(1):19-29.

46 Ling Z, Chen S, Zhang X, Xu F. Exploring crystalline-structural variations of cellulose during alkaline pretreatment for enhanced enzymatic hydrolysis. Bioresour Technol. 2017;224:611-7.

47 Sluiter JB, Ruiz RO, Scarlata CJ. Compositional Analysis of Lignocellulosic Feedstocks. Review and Description of Methods. J Agr Food Chem. 2010;58(16):9043-53. 
Figures

Fig. 1 Enzymatic hydrolysis efficiency of pretreated bamboo residues by three organic reagents (a), the relationship between removal yield of lignin and accessibility,(b), the relationship between pretreated bamboo residues by three organic reagents and hydrophobicity (c), and zeta potential (d) of substrates. Fig. 2 Effects of different lignin addition extracted from acid-pretreated bamboo residues on enzymatic hydrolysis of Avicel (a), $20 \%$ surface lignin (b), 40\% surface lignin and (c), $40 \%$ milled wood lignin; The relationship between enzymatic digestibility of Avicel and (d), contact angel and (e), molecular weight. Fig. 3 The dynamic process of different surface lignin and cellulase binding in real time monitored by SPR technology (a), Dio-SL (b), Eth-SL and (c), THF-SL; The dynamic process of different milled wood lignin and cellulase binding in real time monitored by SPR technology (d), DAP-MWL (e), Dio-MWL (f), Eth-MWL and (g), THF-MWL.

Fig. 4 The relationship between enzymatic hydrolysis efficiency of pretreated bamboo residues by three organic reagents and $(\mathbf{a}), \mathbf{K}_{\mathrm{D}}$ of milled wood lignin (b), $K_{D}$ of surface lignin. The relationship between $K_{D}$ of lignin and (c), enzymatic hydrolysis efficiency of Avicel, (d), Molecular weight and (e), Contact angel. 
Figures
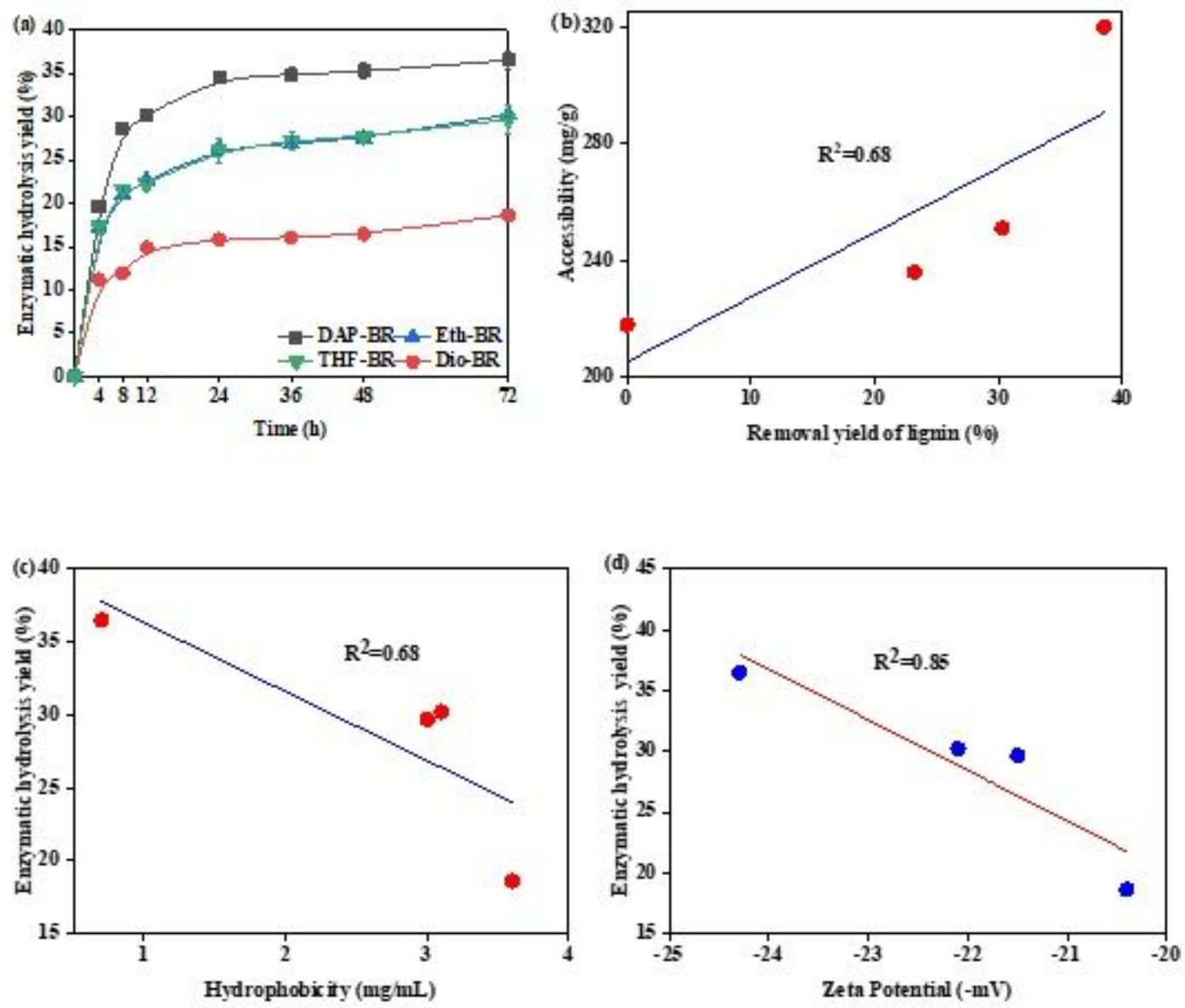

\section{Figure 1}

Enzymatic hydrolysis efficiency of pretreated bamboo residues by three organic reagents (a), the relationship between removal yield of lignin and accessibility,(b), the relationship between pretreated bamboo residues by three organic reagents and hydrophobicity (c), and zeta potential (d) of substrates. 

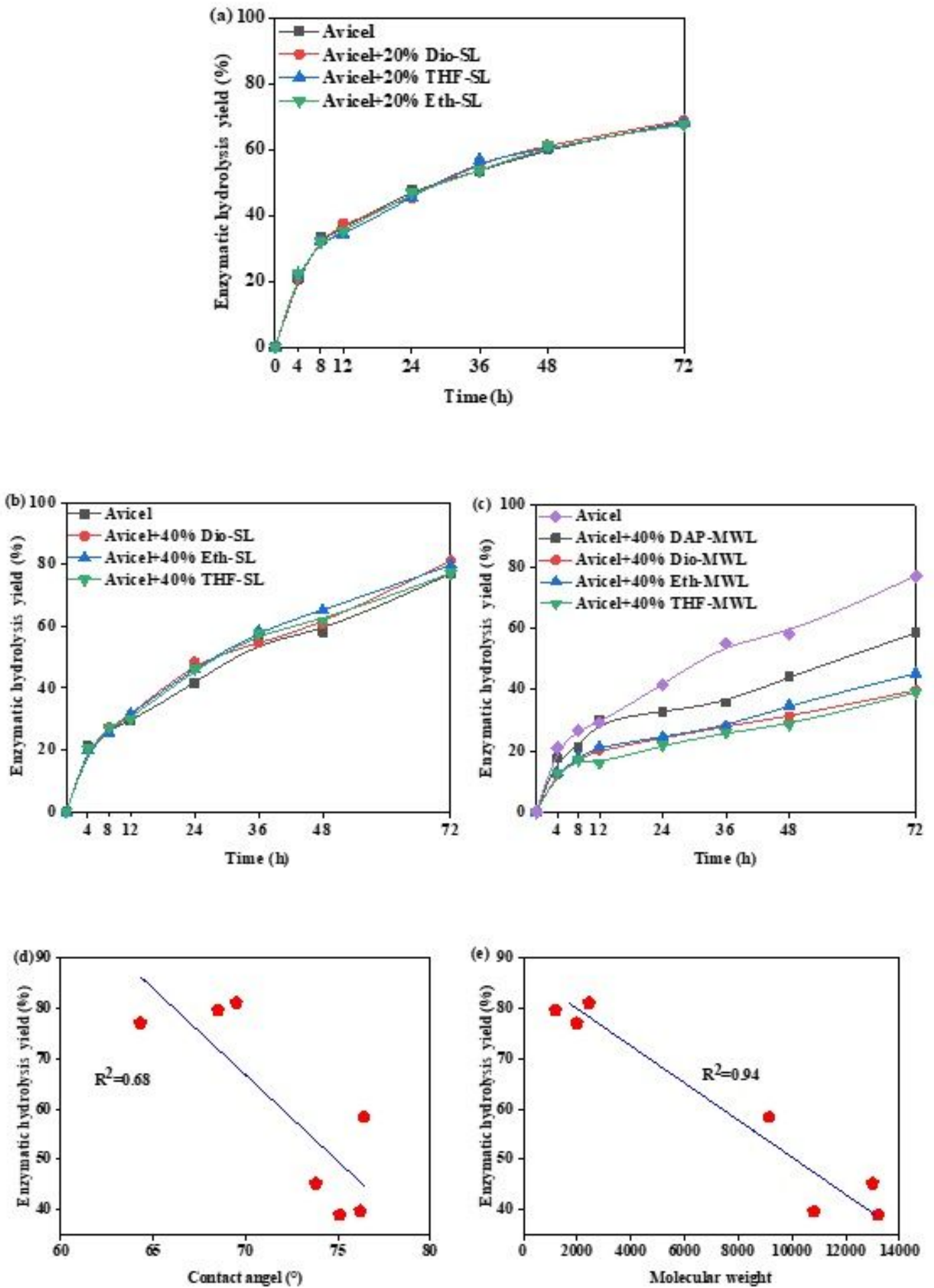

Figure 2

Effects of different lignin addition extracted from acid-pretreated bamboo residues on enzymatic hydrolysis of Avicel (a), 20\% surface lignin (b), $40 \%$ surface lignin and (c), $40 \%$ milled wood lignin; The relationship between enzymatic digestibility of Avicel and (d), contact angel and (e), molecular weight. 

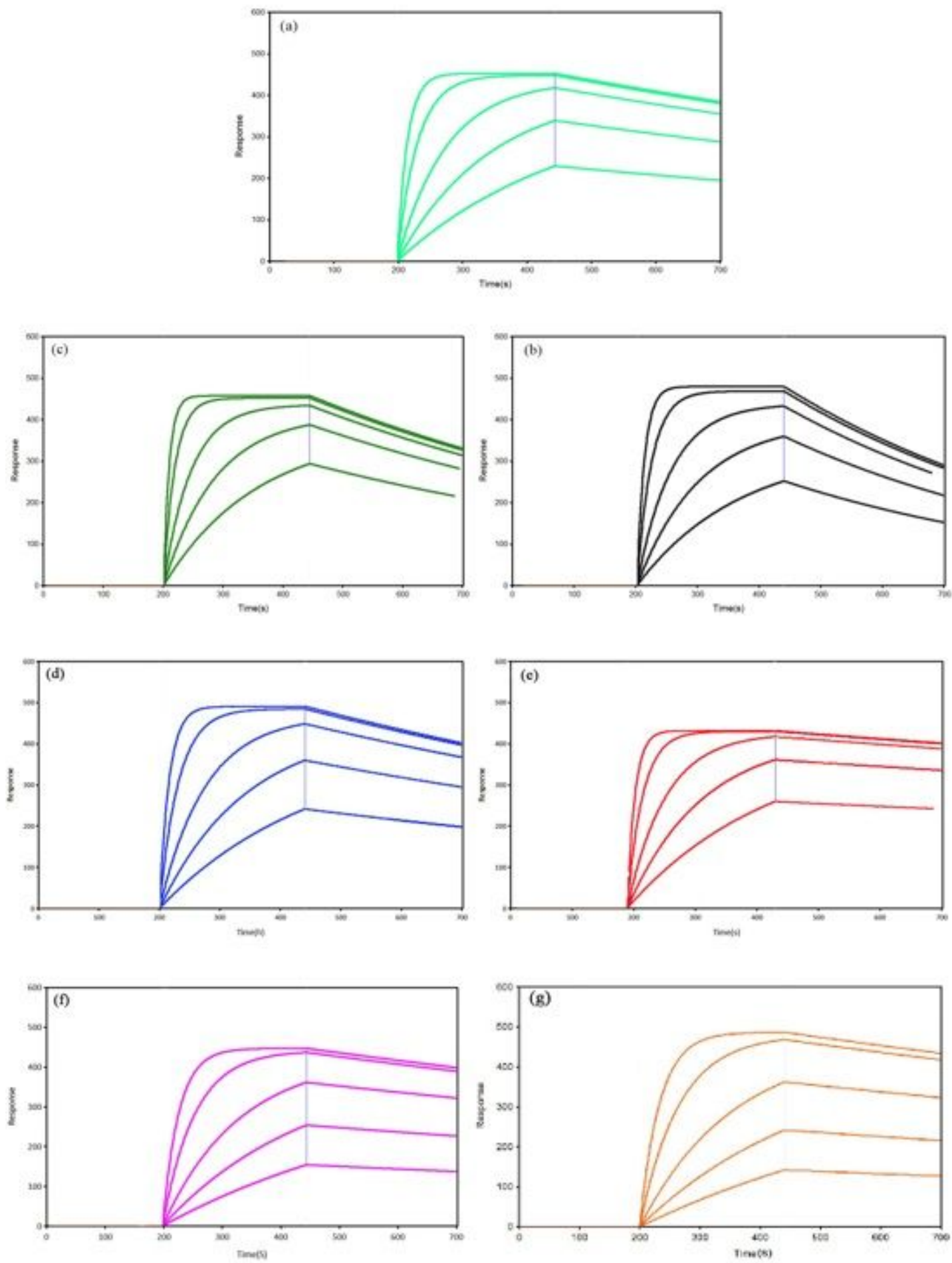

Figure 3

The dynamic process of different surface lignin and cellulase binding in real time monitored by SPR technology (a), Dio-SL (b), Eth-SL and (c), THF-SL; The dynamic process of different milled wood lignin and cellulase binding in real time monitored by SPR technology (d), DAP-MWL (e), Dio-MWL (f), Eth-MWL and $(\mathrm{g})$, THF-MWL. 

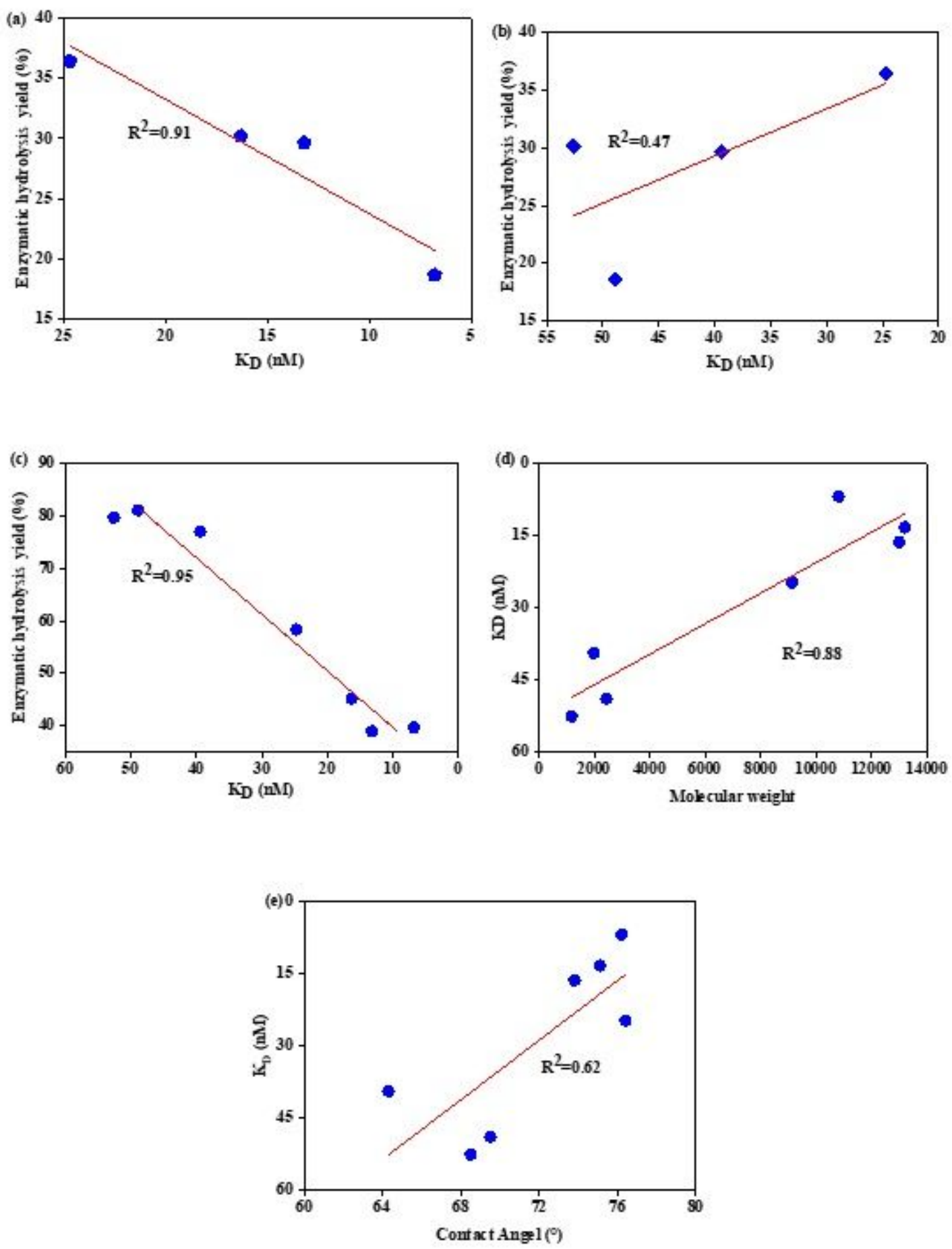

\section{Figure 4}

The relationship between enzymatic hydrolysis efficiency of pretreated bamboo residues by three organic reagents and (a), KD of milled wood lignin (b), KD of surface lignin. The relationship between KD of lignin and (c), enzymatic hydrolysis efficiency of Avicel, (d), Molecular weight and (e), Contact angel.

\section{Supplementary Files}


This is a list of supplementary files associated with this preprint. Click to download.

- Additionalfile1.docx

- Additionalfile2.doc 